Relations industrielles

Industrial Relations

\title{
La légalité des clauses de sécurité syndicale doit-elle être encore remise en cause?
}

\section{Fernand Morin}

Volume 24, numéro 4, 1969

URI : https://id.erudit.org/iderudit/028074ar

DOI : https://doi.org/10.7202/028074ar

Aller au sommaire du numéro

Éditeur(s)

Département des relations industrielles de l'Université Laval

ISSN

0034-379X (imprimé)

1703-8138 (numérique)

Découvrir la revue

Citer cet article

Morin, F. (1969). La légalité des clauses de sécurité syndicale doit-elle être encore remise en cause? Relations industrielles / Industrial Relations, 24(4), 789-795. https://doi.org/10.7202/028074ar

Tous droits réservés (C) Département des relations industrielles de l'Universite Laval, 1969
Ce document est protégé par la loi sur le droit d'auteur. L'utilisation des services d’Érudit (y compris la reproduction) est assujettie à sa politique d'utilisation que vous pouvez consulter en ligne.

https://apropos.erudit.org/fr/usagers/politique-dutilisation/ 


\title{
LA LÉGALITÉ DES CLAUSES DE SÉCURITÉ SYNDICALE DOIT-ELLE ÊTRE ENCORE REMISE EN CAUSE ?
}

\author{
FERNAND MORIN
}

Après la fameuse décision de la Cour suprême dans l'affaire Paquet (1) et celle de la Cour d'appel dans Hôpital St-Luc (2), plusieurs personnes soutenaient que le débat sur la légalité des clauses de sécurité syndicale était clos. Quelques décisions récentes nous incitent à réouvrir ce dossier. Quels sont les droits du salarié qui refuse d'adhérer au syndicat protégé par une telle clause? Si un syndicat expulse de ses rangs un salarié, l'employeur doit-il, en raison de ces clauses, le mettre à pied? Dans la mesure où les différentes clauses de sécurité syndicale sont légales, c'est-à-dire dans la mesure où elles peuvent être imposées comme condition de travail à la convention collective, ne devons-nous pas en accepter, par la suite, leurs effets?

Parce qu'il subsiste encore des doutes quant à la légalité de certaines de ces clauses (celles qui contraignent un salarié d'adhérer à un syndicat donné), le débat n'est pas parfaitement terminé. Nous illustrerons ce point à l'aide de deux décisions: l'une de la Cour d'appel, l'autre de la C.R.T. .

\section{DÉCISION DE LA COUR D'APPEL (3)}

A la suite d'une négociation infructueuse, les parties s'entendent pour soumettre le différend à l'arbitrage. La sentence arbitrale qui en résulte et qui tient lieu de convention collective selon l'article 81 C.t., impose une clause de sécurité syndicale mixte. $M$. le juge Choquette résume ainsi la portée de cette disposition:

«La sentence attaquée rend cette retenue obligatoire. Elle oblige la demanderesse à retenir du salaire de ses employés l'équivalent de la cotisation mensuelle en plus du droit d'entrée pour les nouveaux employés. Elle n'oblige cependant aucun employé à devenir membre du syndicat. Ceux qui le sont déjà ou qui le deviendront dans l'avenir ne pourront toutefois mettre fin à leur affiliation sans un avis à cet effet donné durant la période commençant soixante jours et se terminant trente jours avant l'expiration de la nouvelle convention ». (4)

\footnotetext{
(1) Syndicat catholique des employés de magasins de Québec Inc. c. La Compagnie Paquet Ltée, 1959 R.C.S. 206.

(2) Building Service Employees' International Union c. Hôpital St-Luc et Jewish General Hospital, 1960 B.R. 875, confirmé par 1962 R.C.S. 776.

(3) Montreal Children's Hospital c. Le Syndicat national des employés de Montreal Children's Hospital et l'Heureux et autres, 1969 B.R. 341.

(4) 1969 B.R. 343.
} 
L'employeur conteste la validité d'une telle disposition imposée par sentence arbitrale au moyen de trois arguments:

$I^{\text {er }}$ argument: l'affaire Paquet ne peut s'appliquer puisque la Cour suprême s'était prononcée sur une convention collective librement consentie; dans le présent cas, cette disposition est imposée à l'employeur. Cețe première objection n'est pas retenue parce que selon l'article 81 C.t., cette sentence arbitrale de différend tient lieu de convention collective pour les parties:

«Il s'ensuit que la sentence arbitrale attaquée doit être tenue pour l'expression légale de la volonté des parties; partant, que les clauses 9.01 et 9.02 ont le même effet que si on les trouvait dans une convention collective.

La Cour suprême ayant décidé, dans l'affaire Paquet, que la retenue stipulée dans une convention collective était une condition de travail (condition of employment) et la sentence en litige ayant l'effet d'une convention collective, il n'était pas nécessaire pour les arbitres de préciser que la retenue était stipulée à titre de condition de travail ou as a condition of employment $\gg$. (5)

$2^{e}$ argument: dans l'affaire Hôpital St-Luc, la Cour d'appel et la Cour suprême ${ }^{(6)}$ ont déjà reconu qu'une sentence arbitrale de différend ne pouvait imposer une telle condition de travail. M. le juge Choquette soutient qu'il n'est pas lié par ces deux décisions puisqu'il s'agissait alors d'un cas où on voulait imposer à l'employeur de payer un montant équivalent à la cotisation syndicale pour les salariés qui refusaient de payer eux-mêmes ce montant.

$3^{e}$ argument: une telle disposition serait contraire à l'article 12 C.t. qui prohibe l'usage «... d'intimidation ou de menaces pour amener quiconque à devenir membre, à s'abstenir de devenir membre ou à cesser d'être membre d'une association de salarié...». Ce troisième argument ne convainc pas davantage $M$. le juge Choquette:

«La retenue obligatoire stipulée dans une convention collective ou dans la sentence qui en tient lieu ne saurait constituer de «l'intimidation» ou des «menaces» pour amener les salariés «à signer»... une autorisation de retenir un montant sur leur salaire comme cotisation» (art. 12 du Code du travail). Les salariés sont censés avoir tous consenti à cette retenue par la voix de leur représentant collectif, le syndicat accrédité. D'ailleurs, rien dans la sentence n'oblige les employés à signer une autorisation quelconque. La retenue est obligatoire avec ou sans autorisation ». (7)

(5) ibid. p. 345 .

(6) Op. Cit., supra note (2).

(7) ibid. pp. 345 et 346 . 
Enfin, M. le juge Choquette reconnaît, il s'agit d'un élément nouveau, la légalité de la clause du maintien d'affiliation:

«... C'est ce qu'on appelle la clause du maintien d'affiliation. Le fait que la clause 9.04 soit stipulée « as a condition of employment » n'a rien d'illégal. Pour les motifs que j'ai indiqués dans l'examen des clauses 9.01 et 9.02, pareille clause ne saurait constituer une menace ou intimidation ni un refus arbitraire d'employer une personne au sens des articles 12 et 13 du Code du travail....»(8)

D'autre part, M. le juge Owen nous fait part de son opinion au sujet de la décision de la Cour suprême dans l'affaire Paquet:

«Although I am still of the opinion that the final judgment in the Paquet case should not be followed, this does not appear to be an appropriate case in which to reopen the question as to the meaning of the term «condition of employment»». (9)

\section{Commentaires}

L'approche de la Cour d'appel est bien différente de celle qu'elle adopta en 1958. M. le juge Choquette n'a pas tenté de définir à nouveau le sens de l'expression "condition de travail » ni non plus, de reprendre une recherche à savoir s'il existe un quelconque mandat présumé en faveur du syndicat. La décision de la Cour suprême dans l'affaire Paquet a suffi à donner ou pour trouver réponse aux trois objections de l'employeur. Depuis 1958, il faut reconnaître que le législateur a aussi donné quelques éléments de réponse au sujet de la légalité de certaines clauses de sécurité syndicale. Selon l'article 38 du Code du travail, l'employeur a maintenant l'obligation, sans même qu'il y ait de convention collective à ce sujet, d'effectuer le précompte des cotisations syndicales sur la simple demande d'un syndiqué. Aussi, la Loi des relations de travail dans l'industrie de la construction (18 décembre 1968) édicte à l'article 28, para. 2 que «le décret doit aussi contenir des dispositions concernant la sécurité syndicale, y compris le précompte des cotisations, ... ». Ces éléments nouveaux empêchent, pour le moins, la reprise du débat sur le principe même de ces clauses de sécurité syndicale ${ }^{(10)}$. Il faut maintenant distinguer celles qui sont légales de celles qui ne le sont pas encore.

La présente décision de la Cour d'appel porte non seulement sur les clauses de retenue des cotisations mais aussi, sur une clause de maintien d'affiliation. Pour cette raison, pouvons-nous soutenir maintenant que toutes les clauses de sécurité syndicale sont légales? Est-il logique, en s'inspirant de l'affaire Montreal Children's Hospital, de prétendre que

(8) ibid. p. 346.

(9) ibid. p. 347.

(10) A titre d'employeur, le gouvernement a accepté une clause de retenue obligatoire des cotisations syndicales ou l'équivalent (formule Rand). 
le même principe s'appliquera dorénavant aux dispositions relatives à l'adhésion syndicale obligatoire? Nous ne le croyons pas parce qu'il existe une nette distinction entre les clauses relatives au maintien d'affiliation et les clauses d'atelier syndical parfait ou d'atelier syndical fermé. En effet, la clause du maintien d'affiliation dont la légalité vient d'être reconnue, oblige seulement de conserver pour un temps une adhésion librement et personnellement donnée par un salarié. La lecture des notes de $\mathrm{M}$. le juge Choquette nous font bien comprendre que c'est sur ce fondement de liberté initiale et d'accord volontaire et personnel du salarié que l'on a reconnu la légalité de la clause du maintien d'affiliation. Nous croyons que la Cour d'appel pourrait décider d'une façon tout à fait différente dans le cas où il s'agit d'une clause contraignant le salarié d'adhérer à tel syndicat ${ }^{(11)}$. La lecture des notes de $\mathrm{M}$. le juge Choquette nous le laisse bien entrevoir. Ainsi, lorsqu'il résume la portée de la présente clause de sécurité syndicale, il prend bien soin de dire: «... elle n'oblige cependant aucun employé à devenir membre du syndicat...» (page 343). Puis, peu après, il nous dira:

\begin{abstract}
«... Quant à la clause 9.02, je souscris à ce commentaire du premier juge: "J'avoue qu'il aurait été plus prudent dans l'article 9.02 d'employer de nouveau le mot «équivalent» au sujet des droits d'entrée ou d'initiation, mais je me refuse à croire que les mis en cause ont voulu par là imposer aux nouveaux employés l'obligation de devenir membres de la défenderesse...» (page 345).
\end{abstract}

Cette dernière observation que nous avons souligné (italique) n'estelle pas suffisamment révélatrice de la position de ces tribunaux face à une disposition contraignant l'adhésion à un syndicat donné ?

Quelle que soit l'approche personnelle que l'on puisse adopter à ce sujet ${ }^{(12)}$, il n'en demeure pas moins que la légalité de certaines de ces clauses, particulièrement celle forçant l'adhésion syndicale pour obtenir ou conserver son emploi peut encore être remise en cause. Cette incertitude peut ne pas toujours être bénéfique pour le syndicat, pour le salarié et même pour l'employeur comme nous l'illustrerons maintenant au moyen d'une récente décision de la C.R.T. .

DÉCISION DE LA C.R.T. ${ }^{(13)}$

A la suite de son expulsion du syndicat, un salarié est congédié par son employeur. Pour cette raison, il formule une plainte à la C.R.T.

(11) Des nuances devront être faites dans le cas d'une convention collective servant de base à un décret pour l'industrie de la construction : art. 28 , para. 2 .

(12) Une rétrospective de ces différentes approches fut donnée par MORIN, F., «Sécurité syndicale et les droits du travail au Québec 》dans Revue du Barreau, vol. 21, p. 449. M. le juge Jean-Charles Simard, président d'un tribunal du travail de différends, fit également une étude du statut juridique des clauses de sécurité syndicale dans l'affaire la Commission scolaire régionale 'Chauveau et autres c. l'Association des enseignants du comté de Québec in : 1968 R.D.T. 295 et s. 
en vertu de l'article 14 C.t. . L'employeur soutient qu'il ne peut s'agir d'un congédiement pour activités syndicales et qu'il n'a fait que respecter la clause d'atelier syndical fermé de la convention collective rédigée comme suit:

«L'employeur s'engage, au nom de tous et chacun de ses membres, à n'employer, pour l'exécution de tous ses travaux, que les ouvriers qui sont membres en règle du Conseil national catholique des métiers de la construction des Cantons de l'Est».

Après cette expulsion, les officiers du syndicat demandèrent à l'employeur de congédier ce salarié en lui faisant part de la résolution suivante:

«Que les membres du comité exécutif du Local 825 de l'Union Internationale, ainsi que l'agent d'affaires du même local, soient exclus du syndicat, conformément à l'article 13 , para. B. et $\mathrm{D}$ de la Constitution du Syndicat, pour préjudice grave au syndicat et propagande en faveur d'association opposée aux principes sociaux du Syndicat $\gg$.

L'employeur a soutenu que si les salariés congédiés «... réglaient leur statut avec le syndicat, il les reprendrait à son emploi et que, s'il ne les a pas encore repris, c'est parce qu'ils n'ont jamais réglé leur cas avec le syndicat; . . . > (14)

Compte tenu de ces données, la C.R.T. rejette la plainte: "Considérant qu'il appert que le requérant n'a pas été congédié par l'intimé à cause de l'exercice par ce salarié d'un droit lui résultant du Code, mais bien par suite de l'application de l'article 6(a) de ladite convention collective ».

\section{Commentaires:}

Il ne s'agit pas de savoir si la C.R.T. eut raison de rejeter cette plainte. Nous ne croyons pas utile aussi d'établir si le syndicat était justifié d'expulser ce salarié. Il nous suffira d'abord de souligner que l'affaire Cabana illustre bien que la question de la légalité de certaines clauses de sécurité syndicale ne constitue pas un simple problème académique. Aux Etats-Unis et dans la plupart des provinces canadiennes, le législateur a pris une position qui est relativement claire: l'atelier syndical fermé est prohibé et il suffit souvent de verser régulièrement un montant équivalant à la cotisation syndicale pour qu'un salarié puisse conserver

(13) Marcel Cabana c. Donat Délisle \& Fils Limitée et Association Unie des compagnons et apprentis de l'industrie de la plomberie et de l'ajustage et en tuyauterie des Etats-Unis et du Canada, local 825. In : Décision de la Commission des relations du travail, avril 1969, p. 305.

(14) ibid. p. 308. 
son emploi (15). Ces deux dernières modalités ne règlent pas toutes les questions que soulève un tel problème mais, elles confèrent à tous une protection minimum. Au Québec, le législateur refuse jusqu'ici de prendre position ${ }^{(16)}$, ce qui fait qu'un salarié doit souvent maintenir son adhésion s'il veut conserver son emploi. Sous un régime d'atelier syndical parfait ou fermé, un salarié risque beaucoup s'il entend changer d'allégeance syndicale. S'il le fait ouvertement, il perdra alors son emploi (17). S'il demeure pour un temps membre du premier syndicat tout en adhérant à un autre syndicat, il risque que l'on découvre son jeu et qu'on l'expulse c'est-à-dire, dans un deuxième temps, qu'il soit également congédié. L'importance de cette relation entre l'adhésion à un syndicat particulier et le maintien de son emploi en raison de clause semblable fut déjà mise en relief par M. le juge Fauteux:

«Union Mark for members of the working classes is now a requisite to obtain work. This requisite is clearly essential in cases of closed shop and virtually so in nearly all of the other cases. In the words of Mr. Justice Rand in Orchard et al. v. Tunney:

Membership is the badge of admission and continuance and, vis-à-vis the employer, to remove the badge is directly and immediately to defeat the right.

These are facts that are now given effective recognition in labour and industrial laws where labour relations, labour conditions, collective agreements and industrial peace are, amongst other matters, dealt with. The right here involved is the right which respondent shares with any other member of the working classes to maintain himself in a position to obtain work and, for all practical purposes, it is the right to earn his living. And those who exercise a control over union membership hold, towards the working classes, a position which the law effectively raises above the level of a merely private nature. 》(18)

Je ne préconise pas la prise de mesure de protection en faveur du salarié qui désire s'isoler et agir en franc-tireur: ignorons-le tout comme lui

(15) Opus cite supra note (12) aux pages 460 et s.

(16) L'histoire de notre législation de 1924 à 1944 (Loi des syndicats professionnels et Loi de l'extension des conventions collectives) nous révèle que nous avons déjà protégé expressément la liberté de ne pas se syndiquer (aspect négatif de cette liberté) : ces règles sont maintenant abrogées. L'élaboration du Code du travail en 1964 eut été une excellente occasion pour légiférer en cette matière. Un tel silence au lendemain de l'affaire Paquet démontre bien maintenant qu'il s'agit d'une abstention préméditée.

(17) Rappelons que dans l'affaire Hoogendoorn, la Cour suprême n'a même pas discuté de la légalité d'un tel congédiement en raison du refus d'autoriser la retenue de la cotisation syndicale : seule la question du droit d'être entendu fut étudiée. In : Dick Hoogendoorn c. Greening Metal Products and Screening Equipment Company, 1968 R.C.S. 30.

(18) Seafarer's International Union of North America et J. Stern, 1961 R.C.S. 682. Remarquons que dans ces trois cas célèbres (Orchard v. Tunney, Stern et Hoogendoorn), la protection de l'emploi du salarié fut traitée d'une façon indirecte : les trois décisions portent principalement sur des points incidents. 
d'ailleurs. Nous devrions protéger efficacement le salarié qui entend exercer positivement sa liberté syndicale. Il est inadmissible qu'un salarié puisse perdre automatiquement son emploi et sans autre formalité par suite d'un refus d'adhésion ou de son retrait ou de son rejet d'un syndicat donné. Le syndiqué doit également être mis à l'abri des décisions arbitraires ou politiques de quelques officiers ou factions du syndicat. A ce sujet, signalons à nouveau la mesure spéciale prévue à la Loi des relations de travail dans l'industrie de la construction:

«Article 39:

Une association de salariés ne peut exercer des mesures discriminatoires contre un salarié pour la seule raison qu'il adhère à une autre association ou s'abstient d'adhérer à une association. »

«Article 44, para. 2:

L'arbitre peut aussi ordonner à l'association de salariés de reintégrer le salarié dans ses rangs avec le maintien des avantages dont il a été privé illégalement».

Ces conflits entre le salarié et un syndicat peuvent également se produire entre un syndicat et les fédérations, les conseils régionaux et les centrales syndicales. Pour régler ces questions et pour protéger les droits respectifs de ces différents groupes contre toutes tendances impérialistes ou despotiques, nos syndicalistes ressentiront un jour ou l'autre le besoin d'établir un tribunal d'appel constitué de personnes neutres semblables au "Public Review Board International Union, U.A.W. ». Quoiqu'il en soit, ces questions sont des plus complexes comme le reconnaît le professeur Carrothers:

«... The right of the individual employee as against the employer to join a union, the statutory duty of the employer not to participate in union affairs, the common law right of the union to excluse persons from membership, the concept of majority rule in collective bargaining, the power of the union to bargain to make membership a condition of employment, and the power of the union to bargain for exclusive jurisdiction over job performance, can all converge into a confused vortex, placing at times the employee, the union and the employer in difficult and contradictory positions. »(19)

Cependant, la difficulté de ce problème ne justifie certes pas un silence absolu du législateur. Depuis que l'action syndicale ne se limite plus à une revendication à l'avantage exclusif des salariés les mieux nantis, la liberté syndicale et la démocratie syndicale prennent une importance capitale dans l'ordonnancement de notre société.

(19) Carrothers, A.W.R., Collective Bargaining Law in Canada, Butterworths, Toronto, 1965 , p. 190. 